\title{
Nanocrystal assembly for bottom-up plasmonic materials and surface-enhanced Raman spectroscopy (SERS) sensing*
}

\author{
Andrea R. Tao \\ Department of Chemistry, University of California Berkeley, Berkeley, CA 94720, \\ USA and Materials Science Division, Lawrence Berkeley National Laboratory, \\ Berkeley, CA 94720, USA
}

\begin{abstract}
Plasmonic materials are emerging as key platforms for applications that rely on the manipulation of light at small length scales. Sub-wavelength metallic features support surface plasmons that can induce huge local electromagnetic fields at the metal surface, facilitating a host of extraordinary optical phenomena. Ag nanocrystals (NCs) and nanowires (NWs) are ideal building blocks for the bottom-up fabrication of plasmonic materials for photonics, spectroscopy, and chemical sensing. Faceted Ag nanostructures are synthesized using a colloidal approach to regulate nucleation and crystallographic growth direction. Next, new methods of nanoscale organization using Langmuir-Blodgett (LB) compression are presented where one- and two-dimensional assemblies can be constructed with impressive alignment over large areas. Using this method, plasmon coupling between Ag nanostructures can be controlled by varying spacing and density, achieving for the first time a completely tunable plasmon response in the visible wavelengths. Lastly, these assemblies are demonstrated as exceptional substrates for surface-enhanced Raman spectroscopy (SERS) by achieving high chemical sensitivity and specificity, exhibiting their utility as portable field sensors, and integrating them into multiplexed "lab-on-a-chip" devices.
\end{abstract}

Keywords: colloidal synthesis; nanocrystals; nanowires; plasmonics; surface-enhanced Raman spectroscopy; chemical sensing.

\section{INTRODUCTION}

New methods of colloidal synthesis have allowed the study of a diverse range of inorganic nanocrystals (NCs) with controlled sizes, shapes, and surface morphologies. Unlike bulk materials, geometry profoundly affects the physical properties of a material at the nanoscale, such as the polarized light emission of CdSe nanorods [1] or the light-mediated aggregation of triangular Ag prisms [2]. In the case of noble metal nanoparticles, shape dependence is particularly evident. For example, Ag and Au NCs of different shapes possess unique optical scattering responses. Whereas highly symmetric spherical particles exhibit a single scattering peak, anisotropic shapes such as rods [3], triangular prisms [2], and cubes [4] exhibit multiple scattering peaks in the visible wavelengths due to highly localized charge polarizations at corners and edges. The synthesis and assembly of these nanoscale structures dictate the ultimate materials function. As a consequence, a "design-by-synthesis" approach can be used to de-

\footnotetext{
*Pure Appl. Chem. 81, 1-84 (2009). A collection of invited, peer-reviewed articles by the winners of the 2008 IUPAC Prize for Young Chemists.

¥Current address: Institute for Collaborative Biotechnologies, University of California Santa Barbara, Santa Barbara, CA 931065100, USA
} 
velop new materials with tailored properties such as optical scattering, electromagnetic field enhancement, surface chemistry, and catalytic activity.

In this brief review, we present the shape control of metal NCs as a new design paradigm for generating plasmonic materials. Most work in this area has utilized top-down approaches to fabricate metal structures such as hole and bump arrays. Here, colloidal synthesis is advantageous because it can achieve nanoscale materials with exceptional yield and high monodispersity without the limitations imposed by lithography. We first discuss the synthesis of $\mathrm{Ag}$ nanoparticles with well-defined shapes and sizes, focusing on the polyol process where metal salt is reduced in the presence of a stabilizing polymer. Next, we cover assembly techniques that address the problem of hierarchical nanoscale construction over macroscopic areas. Finally, we discuss the application of shaped Ag NCs and their assemblies as platforms for chemical sensors utilizing surface-enhanced Raman spectroscopy (SERS).

\section{SHAPE-CONTROLLED SYNTHESIS}

Because metal nanostructures generate plasmon-mediated evanescent fields near their surfaces when irradiated with light, Ag NCs and nanowires (NWs) are ideal building blocks for rationally designed plasmonic materials. For bottom-up assembly, it is imperative that these colloidal structures are produced in high yield with excellent size and shape uniformity given that the optical properties exhibited by noble metal NCs are dictated by morphology. Furthermore, long-range assembly at the nanoscale often requires sample homogeneity. Thus, the nucleation and growth processes for colloidal NC synthesis must be well controlled. To achieve shape anisotropy at the crystallographic level, molecular capping agents that selectively adsorb to specific crystal planes can be employed. This general strategy involves stabilizing a particular facet through this molecular interaction: growth is prevented on the crystal plane where binding is strong; growth is promoted on the crystal plane where binding is weak. For face-centered cubic (FCC) noble metals, few molecules have been shown to possess selective binding preferences between the lowest energy $\{100\}$ and $\{111\}$ crystal planes. Particularly impressive shape control has been shown with the use of the amine-terminated surfactants, where encouraging a favored growth direction can yield anisotropic rods as well as bipod, tripod, and tetrapod nanostructures [5]. Other synthetic strategies have explored utilizing the molecular recognition of biomolecules such as proteins and nucleic acids, which often contain various sulfide, amide, and carbonyl functional groups [6-8]. The main advantage of these bio-inspired routes is the existence of a huge molecular library, enabling a combinatorial approach to achieving NC shape control [9].

\section{Polyol process}

Monodisperse colloidal solutions of Ag nanostructures have been successfully synthesized using the polyol process, employing the polymer poly(vinyl pyrolidone) (PVP) as a molecular capping agent. First patented in 1985 by Figlarz as a general chemical reaction for producing monodisperse metal powders in a liquid medium [10], the chemical reaction involves the reduction of silver nitrate in a diol solvent at near-reflux temperatures. Xia reported the use of PVP for the formation of Ag NWs [11] and nanocubes [4]. The effect of PVP is easily seen in the formation of NWs, where the polymer adsorbs to the $\{100\}$ facets and restricts radial growth (Fig. 1). Using an adapted procedure, we employed a more viscous solvent and trace amounts of a secondary metal ion to achieve more regulation of nucleation (Fig. 2). By promoting a single, fast nucleation event, we successfully synthesized a variety of NC shapes with uniform sizes: cubes $(\sim 80 \mathrm{~nm})$, truncated cubes $(\sim 120 \mathrm{~nm})$, cuboctahedra $(\sim 150-200 \mathrm{~nm})$, truncated octahedra $(\sim 200-250 \mathrm{~nm})$, and octahedra $(\sim 250-300 \mathrm{~nm})$. Transmission electron microscopy (TEM) confirms that these polyhedra are single crystalline and possess atomically defined facets with sharp edges and corners. Each of these dispersions exhibits sharp plasmon resonances in the visible wavelengths that correspond well with predicted spectra given by electromagnetic theory. For a more detailed description of shape control using the polyol process, see ref. [12]. 

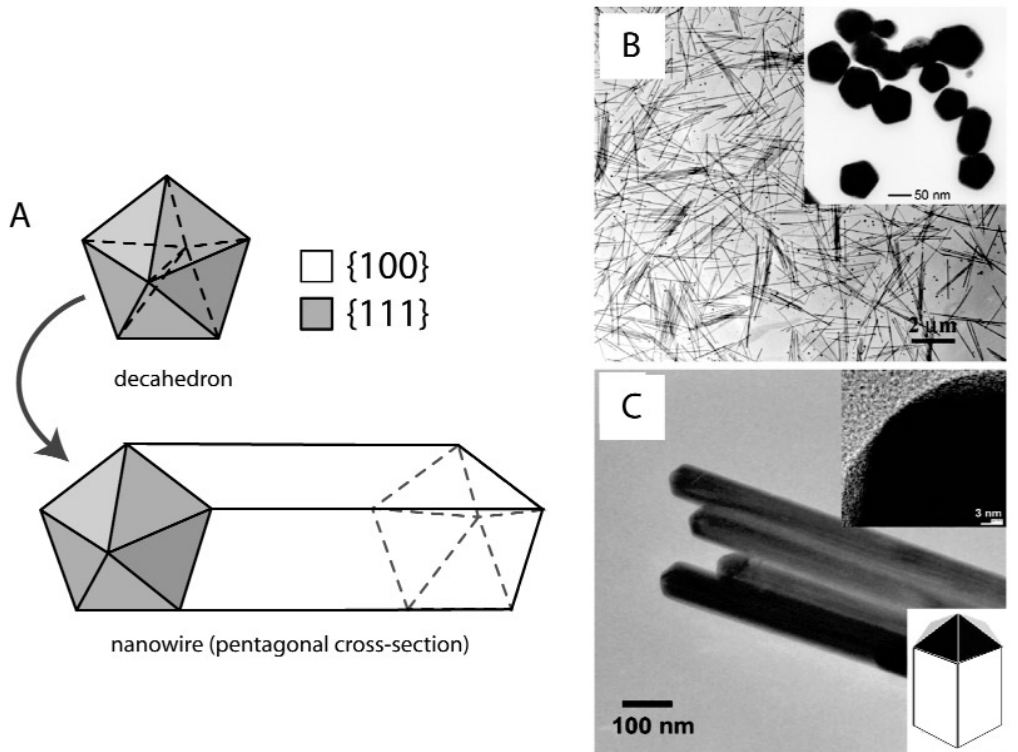

Fig. 1 (A) Schematic depiction of NW growth from a decahedral seed particle. (B) Uniform solution of wires with a microtomed cross-section shown in the inset. (C) TEM image of NWs with a high-resolution image of the wire tip. Adapted from ref. [21].

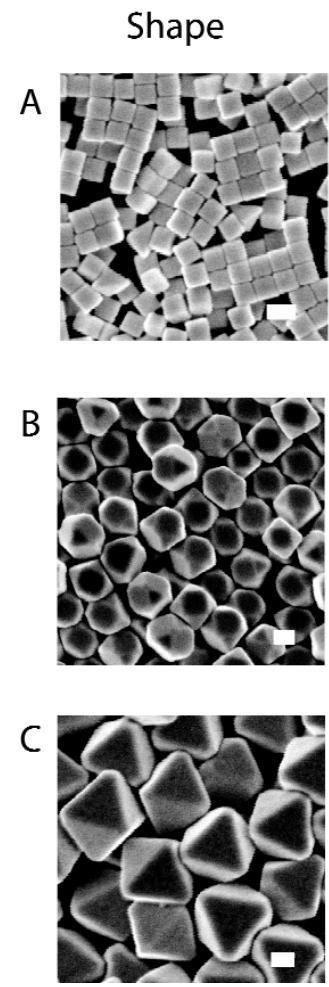

\section{Experiment}
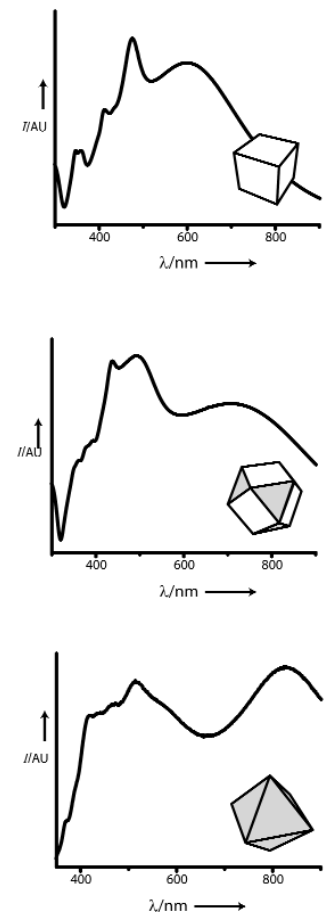

Theory
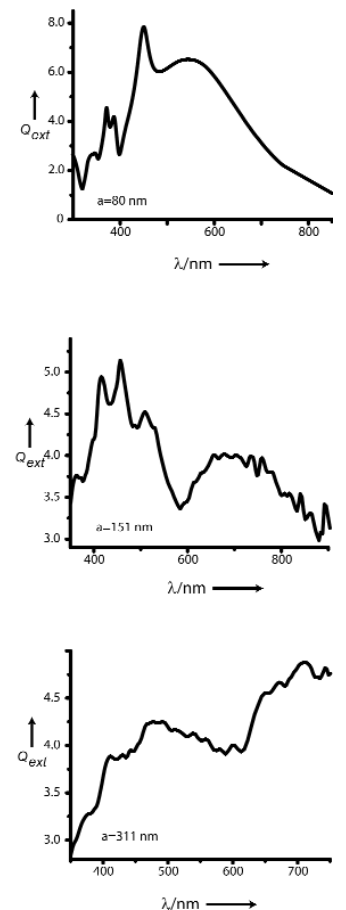

Fig. 2 From left to right: SEM images of Ag NCs, UV-vis spectra of colloidal solutions, and theoretical discrete dipole approximation scattering for $(\mathrm{A})$ cubes, $(\mathrm{B})$ cuboctahedra, and $(\mathrm{C})$ octahedra. Scale bar $=100 \mathrm{~nm}$. In depictions, blue areas represent $\{111\}$ facets, white areas represent $\{100\}$ facets. Adapted from ref. [50]. 


\section{LANGMUIR-BLODGETT ASSEMBLY}

Controlled positioning of nanoparticles using a self-assembly method is highly desirable for its simplicity and compatibility with device integration processes [13]. For plasmonic materials, the bottomup organization of metal nanostructures is largely unexplored. Nanowire (NW) and nanocrystal (NC) assemblies are optimal platforms for plasmonic applications because they promote electromagnetic coupling between metallic NCs, leading to narrow plasmon bands [14] and intense field enhancement in the interstitial spaces between neighboring NCs $[15,16]$. Langmuir-Blodgett (LB) assembly is a general and versatile method of organizing colloidal structures at an air/water interface that enables transfer to an arbitrary substrate, making it a powerful tool for nanoscale assembly. Nanoparticles are dispersed in a volatile solvent that is immiscible in water. This dispersion is then spread dropwise onto a water surface, leaving a water-supported film of isotropically distributed particles. Simple manipulation of this interface can give rise to complex patterns, such as stripes [17] and single-particle lines [18] with tunable widths, particle densities, and spacing (Fig. 3).

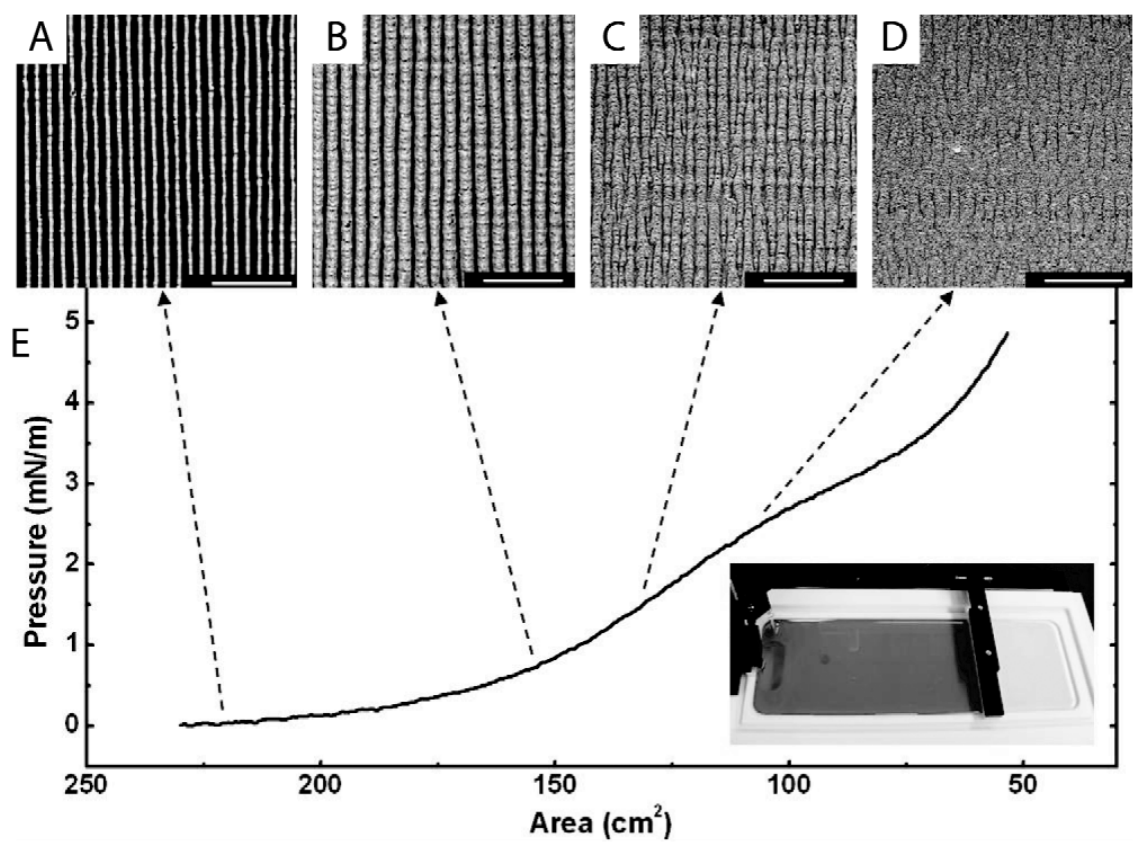

Fig. 3 Stripe formation upon monolayer dewetting. From (A) to (D), substrate dip-coating is performed at increasing monolayer surface pressures, as shown on the isotherm curve in (E). Inset: LB trough with NC monolyer on water surface. Adapted from ref. [17].

LB assembly produces highly ordered monolayers [19], where packing symmetry can be determined by building-block shape. Close-packed arrays are obtained by isothermal compression of the floating NC monolayer using a mechanical barrier (Fig. 4). For anisotropic structures like Ag NWs, the assembly process is a microscopic version of "logs-on-a-river": as the NWs are compressed at the air/water interface, they align side-by-side along their long axis [20]. The compressed Ag NW monolayer exhibits remarkable alignment parallel to the trough barrier. This achieves large-scale nematic ordering with unprecedented NW packing density, allowing the fabrication of high-density nanoscale interconnects and sensor arrays. 

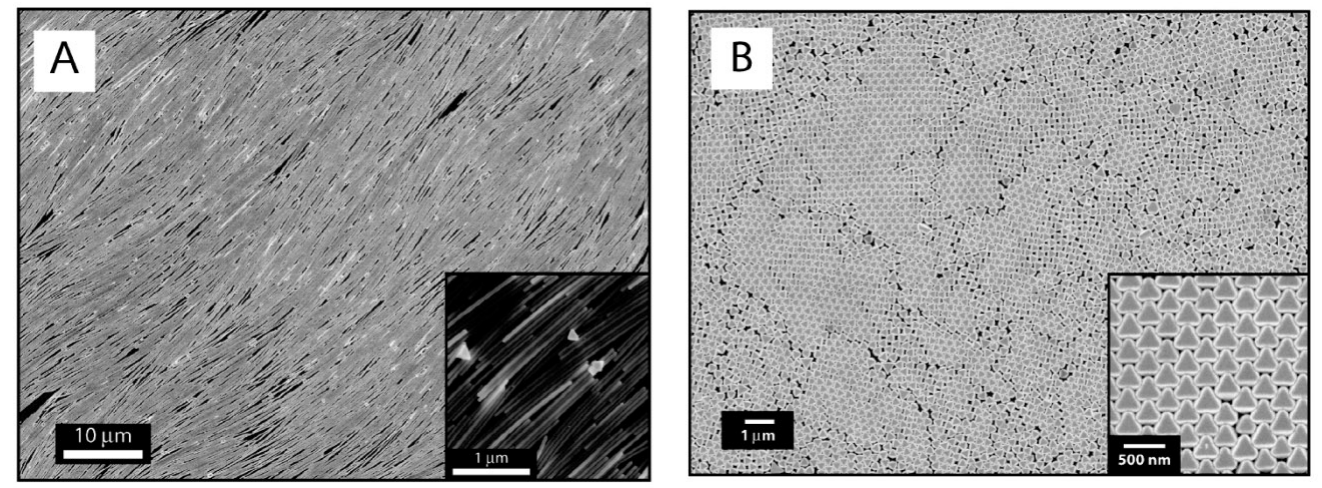

Fig. 4 SEM of dense arrays of (A) Ag NWs and (B) Ag octahedra assembled by LB compression. Insets show a close-up of superlattice packing. Adapted from refs. [20,21].

\section{Tunable plasmonic lattices}

For polyhedral Ag NCs, LB assembly can produce unique 2D superlattices [21]. These assemblies promote electromagnetic coupling between NCs, leading to narrow plasmon bands and intense field enhancement in the interstitial spaces between neighboring particles. A unique feature of the NC monolayer is the ability to tune the plasmon response of the fluid-supported film across the visible range by controlling these electromagnetic interactions (Fig. 5). Because electromagnetic coupling is distancedependent, plasmon resonances can be continuously tuned across the visible range. Figure 5 shows the specular reflectance of a 2D NC superlattice at the air/water interface as it undergoes isothermal compression, with spectra grouped into low $(\Pi \sim 0 \mathrm{mN} / \mathrm{m})$ and high $(0<\Pi<14 \mathrm{mN} / \mathrm{m})$ surface pressure regimes for clarity. The reflectance line shape of the fluid-supported monolayer differs greatly from that of the isotropic colloidal $\mathrm{NC}$ suspension, resulting from the coherent near-field interactions of the ordered array. At maximum surface pressure, the monolayer visually resembles a metallic thin film, displaying a high reflectivity and a characteristic sheen (Fig. 5A). Essentially, the superlattice exhibits the optical properties of a bulk metal surface, which suggests that propagating surface plasmons can be completely engineered by this bottom-up assembly approach. The ability to control the density and strength of collective near-field interactions has important consequences for applications that utilize electromagnetic hot spots, such as SERS or light focusing. 

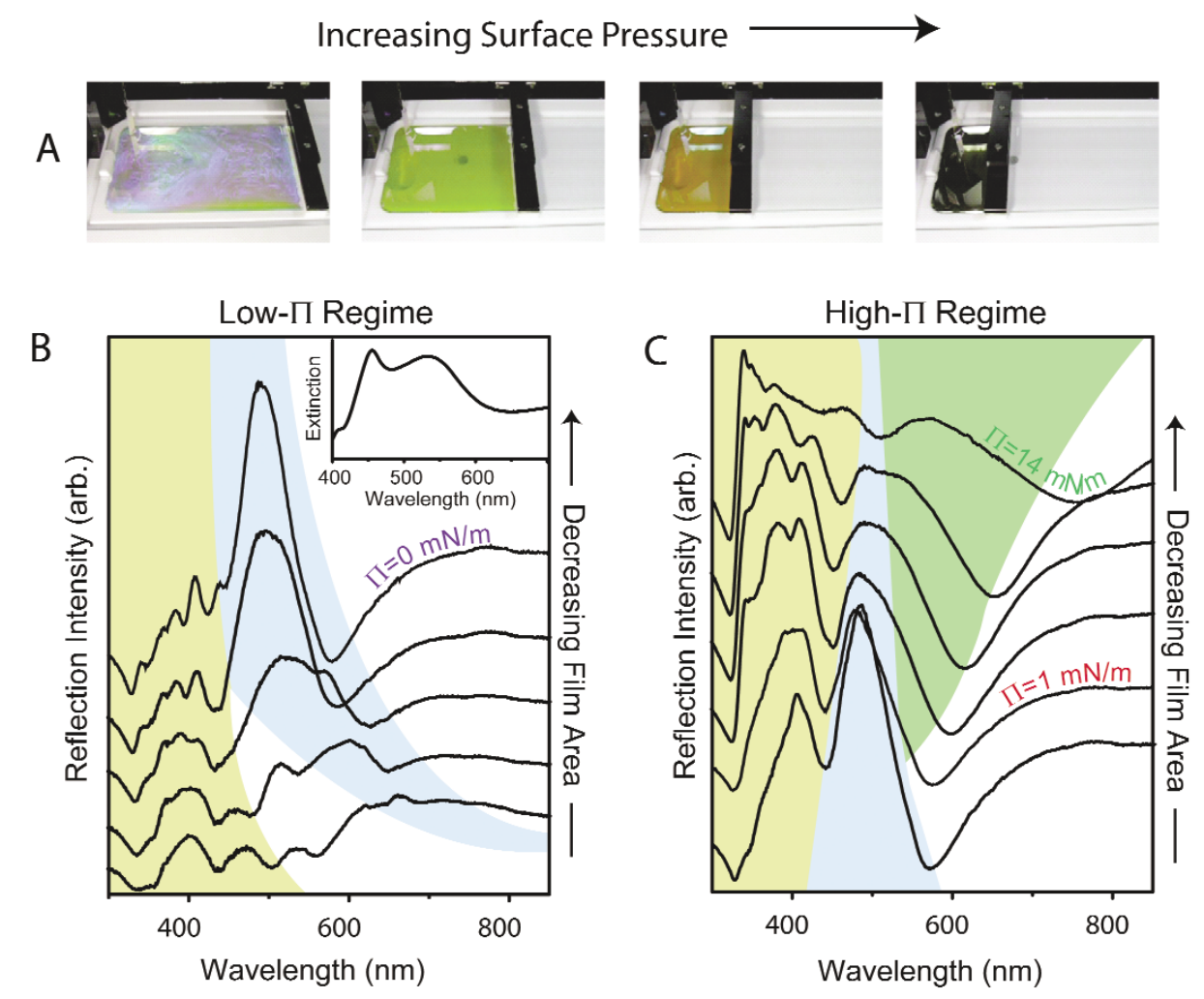

Fig. 5 Tunable plasmonic response of Ag NC monolayers. (A) Images of the NC monolayer as surface pressure is increased. (B) Reflection spectra of the low surface pressure regime showing strong peak due to Bragg scattering. Inset: UV-vis of the colloidal solution. (C) Reflection spectra of the high surface pressure regime, showing evolution of the optical response as plasmon coupling ensues. Adapted from ref. [21].

\section{ANISOTROPIC STRUCTURES FOR SURFACE-ENHANCED RAMAN SPECTROSCOPY}

Shape-controlled NCs and their assemblies are ideal substrates for SERS because their unique morphologies facilitate the intense electromagnetic field amplifications responsible for Raman enhancement. As a sensing strategy, SERS is an advantageous because it provides chemically specific vibrational signatures and is well suited to detection in aqueous environments due to the low Raman cross-section of water [22]. Utilizing the electromagnetic field enhancement associated with nanoscale metal surfaces, SERS intensities have been demonstrated to reach the single-molecule detection limit $[23,24]$. These results have prompted a great deal of interest in SERS sensors for a variety of analytes, including biowarfare agents [25], explosives [20], small biological molecules such as glucose [26], large proteins such as insulin [27], DNA and single nucleotides [28,29], and various hazardous environmental toxins [30]. Typically, values for Raman scattering cross-sections are low and range between $10^{-31}-10^{-29} \mathrm{~cm}^{2} /$ molecule (with the highest values for resonant Raman), compared with fluorescence cross-sections that can reach as high as $10^{-16} \mathrm{~cm}^{2} /$ molecule [31]. SERS overcomes these low scattering cross-sections by introducing a metal particle that can radiate light at the incident and Ramanshifted wavelengths. This radiation occurs with the excitation of a surface plasmon mode, where conduction electrons oscillate in resonance with the incident light wave to produce localized electromagnetic field distributions near the metal surface. These near-field amplifications decay exponentially with a distance $r$ from the metal surface as $r^{-3}$. In practice, molecules must be within $2 \mathrm{~nm}$ of the metal surface to experience significant field enhancement effects [31]. 


\section{SERS-active materials}

Because field enhancement is inherently dependent on the plasmonic properties of the metal particle, choice of metal substrate and excitation wavelength are critical factors that influence observed SERS intensities. The majority of early SERS studies utilized roughened metal electrodes or cold-deposited films, both consisting of a random distribution of feature shapes and sizes [15,32-35]. As details of electromagnetic theory began to emphasize the importance of substrate morphologies, and after it was demonstrated that nanostructured metal surfaces were required for optimizing field enhancement, SERS-active systems were extended to lithographically defined metal islands [36], metal gratings [37], and metal colloids [38-42]. In particular, experiments performed on metal colloids, which often aggregate or self-organize, led to the exploration of electromagnetic hot spots at nanoparticle junctions.

Metal NW films assembled by the LB method have been shown to yield high Raman intensities orders of magnitude higher than traditional substrates (Fig. 6) [20]. Plasmon coupling between NWs is responsible for SERS "hot spots" in the interstitial spaces between adjacent wires, where field localization occurs [43]. SERS intensities for analyte molecules adsorbed onto these wires assemblies are highly dependent on incident polarization. In the familiar "lightning rod effect", intense local electromagnetic fields emanate from points of high curvature, such as the tip of a rod or wire $[44,45]$. For aligned NWs, the local field responsible for the enhanced Raman signal is oriented perpendicular to the long axes of the wires. Here, enhancement arises from pairwise electron microscopy (EM) interactions between adjacent NWs (Fig. 7). In addition, the observed $\cos ^{2} \theta$-dependence is consistent with enhancement stemming from a dipolar plasmon mode. Such experimental observations correlate extremely well with predictions by Garcia-Vidal and Pendry, where SERS enhancements of $10^{7}$ are modeled for modeled aligned Ag half-cylinders [15].
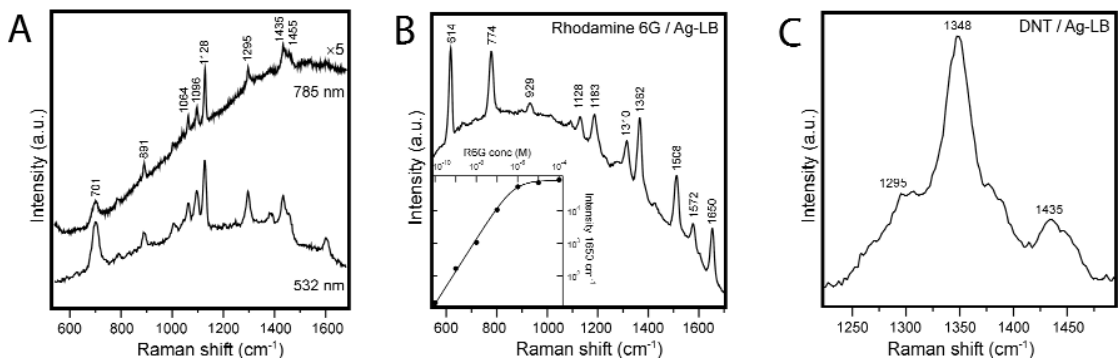

Fig. 6 SERS response from a NW monolayer for (A) a molecular monolayer of hexadecanethiol, (B) adsorbed rhodamine 6G, and (C) adsorbed 2,4-dinitrotoluene, an analyte molecule for explosives. Adapted from ref. [20].

A

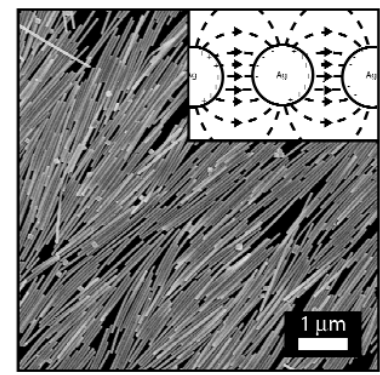

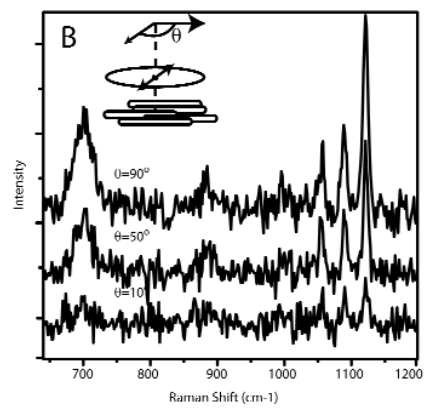

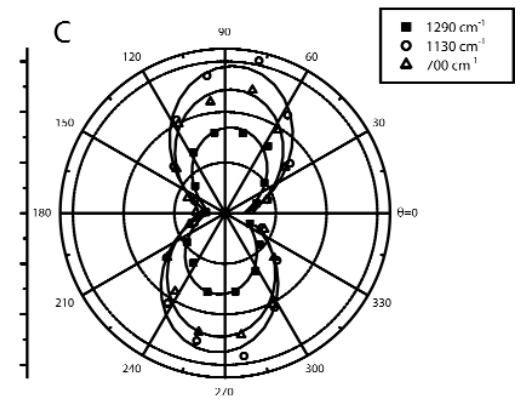

Fig. 7 Polarized SERS response of an NW monolayer. (A) SEM of monolayer and schematice of localized electromagnetic fields between wires. (B) SERS spectrum as a functional of polarization angle for the excitation source. (C) Polarized SERS response for different Raman bands. Adapted from ref. [43]. 


\section{Chemical detection}

With the potential for single-molecule sensitivity, SERS has been widely explored for analytical detection and identification. SERS sensors have been reported for a wide variety of metal substrates capable of detecting analytes ranging from biowarfare [46] agents to chemical explosives [20] to small biological molecules [47]. Most notable are substrates created by nanosphere lithography, where metal is evaporated into the interstitial spaces of a template of close-packed spheres [48]. Assemblies of colloidal nanostructures are advantageous as SERS substrates because they can be optimized to give a high density of active hot spots. For the Ag NW monolayers discussed in the previous section where wires are close-packed into a 2D film, 2,4-dinitrotoluene was detected down to concentrations of $0.7 \mathrm{pg}$. Interestingly, both green $(532 \mathrm{~nm})$ and near-infrared excitation $(785 \mathrm{~nm})$ gave rise to comparable SERS intensities. This effect stems from the electromagnetic coupling of individual Ag wires within the film, which gives rise to a broad plasmon response that is at a maximum around $550 \mathrm{~nm}$ and extends into the near-infrared region. Unlike other substrates, these NW assemblies serve as extremely versatile SERS substrates by allowing excitation over a wide range of frequencies.

In addition, the chemical surface offered by metal colloids can be employed for optimizing SERSbased detection. Close-packed assemblies of octahedral Ag NCs have been utilized as SERS substrates for the identification of arsenic in water down to the low-level concentration of $1.8 \mathrm{ppb}$, well below the toxic limit of $10 \mathrm{ppb}$ established by the World Health Organization [30]. Chronic arsenic poisoning stemming through drinking water is a widespread health risk in developing countries such as Bangladesh, where the skin, lung, and bladder cancers associated with long-term arsenic exposure are responsible for nearly 300000 deaths. NC assemblies serve as a disposable and portable SERS "chip" that is practical for on-site contaminant identification and provides a cost-effective, more sensitive method of low-level arsenic detection than traditional field tests. SERS detection is performed by placing a droplet of the analyte solution directly onto an NC film surface. Adsorbed polymer (PVP) on the surface of the nanoparticles facilitates the binding of both arsenate and arsenite near the Ag surface. The SERS intensities display a linear response for concentrations of arsenate in water between $1.8 \mathrm{ppb}$ and $1.8 \mathrm{ppm}$, allowing quantitative detection and identification between the two species.

\section{Device integration}

On-site field detection of water contaminants, hazardous materials, or even blood-serum content requires a sensing platform that is compact, portable, and for biological applications in particular, multifunctional with the ability to analyze extremely small sample volumes. We developed a microfluidic sensing platform that integrates semiconductor NW waveguides and shaped Ag NCs (Fig. 8) [49]. In this all-photonic sensor, we use $\mathrm{SnO}_{2}$ nanoribbons as the passive optical components in the devices because their higher index of refraction allows efficient waveguiding through the microfluidic devices and analyte solutions. Because the nanoribbons possess sub-200 nm diameters, a substantial amount of the guided optical intensity is exposed to the surrounding matrix and light can then be extracted from the waveguide by introducing a scattering center. In particular, metal nanoparticles provide a high dielectric contrast. We found that $\mathrm{Ag}$ nanocubes $\sim 50 \mathrm{~nm}$ in diameter readily adsorb to the waveguide surface and a single particle can release a large percentage (5-10\%) of the confined optical energy. The scattering intensity from these immobilized nanocubes can be used for SERS whereby monochromatic light $(532 \mathrm{~nm})$ from the waveguide excites surface plasmons. To demonstrate resonate SERS we exposed the nanoribbons to a $100 \mu \mathrm{M}$ solution of rhodamine $6 \mathrm{G}\left(\alpha_{\max }=535 \mathrm{~nm}\right)$ after decorating the waveguide surface with Ag nanocubes. The SERS signal was probed either directly with a diffraction-limited confocal spot focused on the waveguide or via waveguided light. Performing SERS with large $(\sim 500 \mathrm{~nm})$ and small $(\sim 150 \mathrm{~nm})$ diameter nanoribbons confirmed that a stronger Raman signal is achieved when a larger number of particles are scattering in the field of view. The same device configuration can also be used for fluorescence and absorbance measurements, as discussed in ref. [49]. 

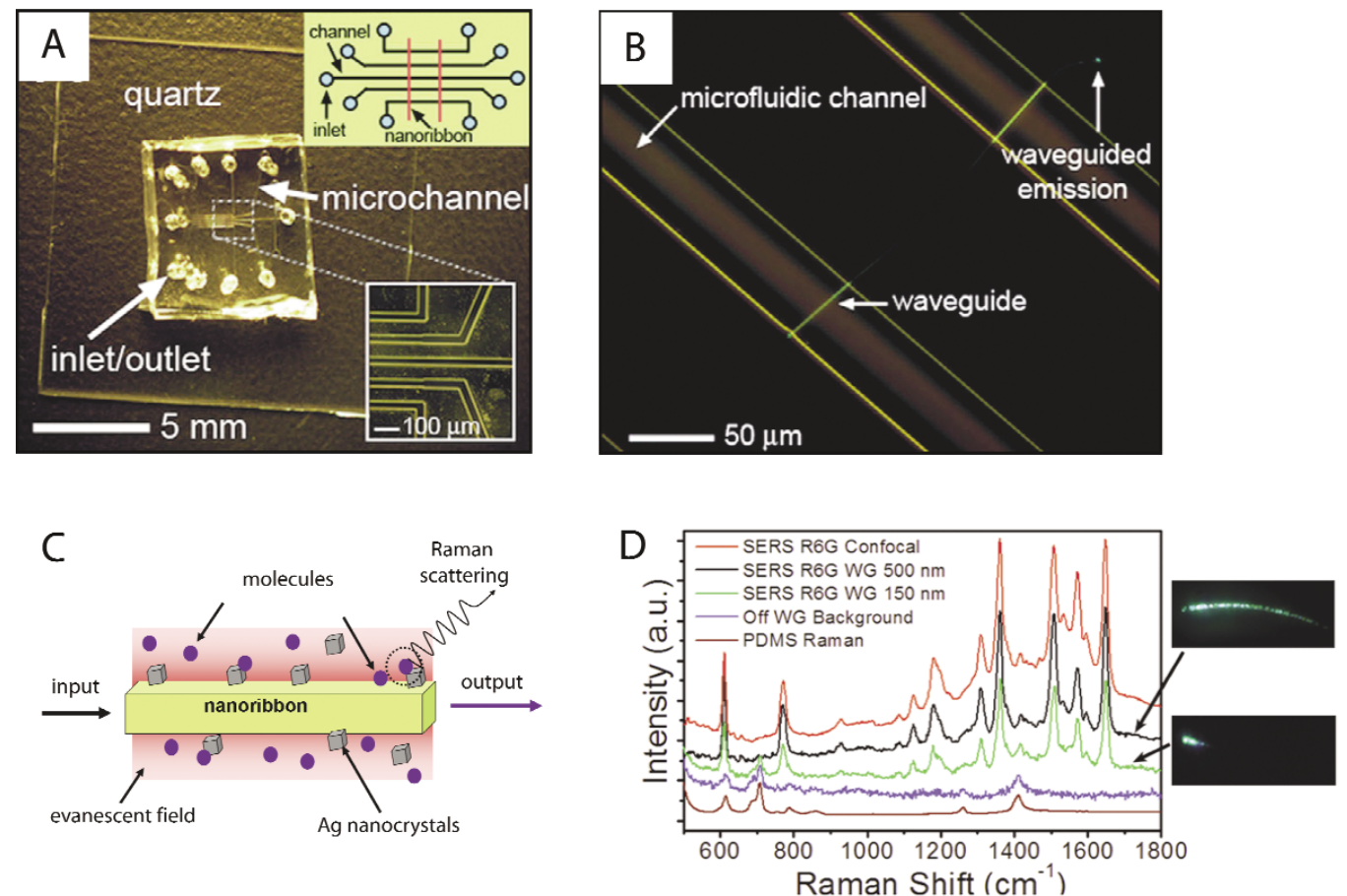

Fig. 8 Lab-on-a-chip device. (A) Microfluidic platform with multiple channels for analyte flow. (B) Close-up of NW waveguides crossing channels. (C) Schematic of SERS sensing strategy, where Ag NCs are attached to the NW and then exposed to the analyte solution. (D) SERS spectra for rhodamine 6G solutions. Adapted from ref. [49].

\section{CONCLUSION}

This short review highlights the exciting prospect of utilizing assembled nanostructures in fundamental and applied studies. The synthesis and assembly of colloidal metal nanostructures are the first steps in pioneering bottom-up plasmonic materials for application in sub-wavelength optics, field-enhanced spectroscopy, and chemical sensing. Using the polyol method, uniform Ag NCs and NWs can be synthesized in high yield and uniformity. Assembly of these colloidal building blocks into hierarchical structures facilitates electromagnetic coupling, giving rise to exceptional SERS activity. The facile integration of these nanostructures into functional devices may facilitate the next generation of chemical sensors which are portable, multiplexed, and cheaply fabricated. Future challenges for such devices include the analysis of complex liquid mixtures such as blood serum, and the spectroscopic monitoring of large biological molecules to give both chemical and spatial information.

\section{ACKNOWLEDGMENTS}

A. R. Tao thanks: the National Science Foundation for a Graduate Research Fellowship and the University of California President's Postdoctoral Fellowship; Martin Mulvihill, Donald J. Sirbuly, and Jiaxing Huang for their collaborative spirit and exceptional work on arsenic sensing, microfluidic device integration, and 1-D assembly projects, respectively; and Prof. Yang for being a thoughtful and supportive mentor throughout my graduate career and beyond. 


\section{REFERENCES}

1. C. B. Murray, D. J. Norris, M. G. Bawendi. J. Am. Chem. Soc. 115, 8706 (1993).

2. R. Jin, Y. Cao, C. A. Mirkin, K. L. Kelly, G. C. Schatz, J. G. Zheng. Science 294, 1901 (2001).

3. Y. Y. Yu, S. S. Chang, C. L. Lee, C. R. C. Wang. J. Phys. Chem. B 101, 6661 (1997).

4. Y. Sun, Y. Xia. Science 298, 2176 (2002).

5. S. Chen, Z. L. Wang, J. Ballato, S. H. Foulger, D. L. Carroll. J. Am. Chem. Soc. 125, 16186 (2003).

6. R. R. Naik, S. J. Stringer, G. Agarwal, S. E. Jones, M. O. Stone. Nat. Mater. 1, 169 (2002).

7. S. Brown, M. Sarikaya, E. Johnson. J. Mol. Biol. 299, 725 (2000).

8. I. A. Banerjee, L. Yu, H. Matsui. Proc. Natl. Acad. Sci. USA 100, 14678 (2003).

9. S. Brown. Nat. Biotechnol. 15, 269 (1997).

10. M. Figlarz, F. Fievet, J.-P. Lagier. U.S. Patent 4539041, Issued 3 Sept. 1985.

11. Y. Sun, B. Mayers, T. Herricks, Y. Xia. Nano Lett. 3, 955 (2003).

12. A. R. Tao, S. Habas, P. Yang. Small 4, 310 (2008).

13. G. M. Whitesides, J. P. Mathias, C. T. Seto. Science 254, 1312 (1991).

14. E. M. Hicks, S. Zou, G. C. Schatz, K. G. Spears, R. P. V. Duyne, L. Gunnarsson, T. Rindzevicius, B. Kasemo, M. Kall. Nano Lett. 5, 1065 (2005).

15. F. J. Garcia-Vidal, J. B. Pendry. Phys. Rev. Lett. 77, 1163 (1996).

16. D. A. Genov, A. K. Sarychev, V. M. Shalaev, A. Wei. Nano Lett. 4, 153 (2004).

17. A. R. Tao, J. Huang, F. Kim, S. Connor, P. Yang. Nat. Mater. 4, 896 (2005).

18. J. Huang, A. R. Tao, S. Connor, R. He, P. Yang. Nano Lett. 6, 524 (2006).

19. C. P. Collier, R. J. Saykally, J. J. Shiang, S. E. Henrichs, J. R. Heath. Science 277, 1978 (1997).

20. A. Tao, F. Kim, C. Hess, J. Goldberger, R. He, Y. Sun, Y. Xia, P. Yang. Nano Lett. 3, 1229 (2003).

21. A. Tao, P. Sinsermsuksakul, P. Yang. Nat. Nanotechnol. 2, 435 (2007).

22. K. Kneipp, H. Kneipp, I. Itzkan, R. R. Dasari, M. S. Feld. Chem. Rev. 99, 2957 (1999).

23. K. Kneipp, Y. Wang, H. Kneipp, L. T. Perelman, I. Itzkan, R. R. Dasari, M. S. Feld. Phys. Rev. Lett. 78, 1667 (1997).

24. S. Nie, S. R. Emory. Science 275, 1102 (1997).

25. C. L. Haynes, C. R. Yonzon, X. Zhang, R. P. V. Duyne. J. Raman Spectrosc. 36, 471 (2005).

26. K. E. Shafer-Peltier, C. L. Haynes, M. R. Glucksberg, R. P. V. Duyne. J. Am. Chem. Soc. 125, 588 (2002).

27. V. P. Drachev, M. D. Thoreson, E. N. Khaliullin, V. J. Davisson, V. M. Shalaev. J. Phys. Chem. B 108, 18046 (2004).

28. K. Kneipp, H. Kneipp, V. B. Kartha, R. Manoharan, G. Deinum, I. Itzkan, R. R. Dasari, M. S. Feld. Phys. Rev. E 57, R6281 (1998).

29. L. R. A. D. L. S. Tuan Vo-Dinh. J. Raman Spectrosc. 33, 511 (2002).

30. M. M. Mulvihill, A. Tao, K. Benjauthrit, P. Yang. Angew. Chem., Int. Ed. 47, 6456 (2007).

31. K. Kneipp, H. Kneipp, I. Itzkan, R. R. Dasari, M. S. Feld. J. Phys.: Condens. Matter 14, R597 (2002).

32. E. V. Albano, S. Daiser, G. Ertl, R. Miranda, K. Wandelt, N. Garcia. Phys. Rev. Lett. 51, 2314 (1983).

33. T. H. Wood, D. A. Zwemer, C. V. Shank, J. E. Rowe. Chem. Phys. Lett. 82, 5 (1981).

34. X. M. Yang, K. Ajito, D. A. Tryk, K. Hashimoto, A. Fujishima. J. Phys. Chem. 100, 7293 (1996).

35. D. D. Tuschel, J. E. Pemberton, J. E. Cook. Langmuir 2, 380 (1986).

36. T. R. Jensen, M. D. Malinsky, C. L. Haynes, R. P. van Duyne. J. Phy. Chem. B 104, 10549 (2000).

37. A. Wirgin, A. A. Maradudin. Prog. Surf. Sci. 22, 1 (1986).

38. A. M. Michaels, J. Jiang, L. Brus. J. Phys. Chem. B 104, 11965 (2000).

39. R. G. Freeman, K. C. Grabar, K. J. Allison, R. M. Bright, J. A. Davis, A. P. Guthrie, M. B. Hommer, M. A. Jackson, P. C. Smith, D. G. Walter, M. J. Natan. Science 267, 1629 (1995). 
40. Y. Lu, G. L. Liu, L. P. Lee. Nano Lett. 5, 5 (2005).

41. A. M. Michaels, M. Nirmal, L. E. Brus. J. Am. Chem. Soc. 121, 9932 (1999).

42. H. Wang, C. J. Levin, N. J. Halas. J. Am. Chem. Soc. 127, 14992 (2005).

43. A. R. Tao, P. Yang. J. Phys. Chem. B 109, 15687 (2005).

44. E. Hao, G. C. Schatz. J. Chem. Phys. 120, 357 (2004).

45. J. Jiang, K. Bosnick, M. Maillard, L. Brus. J. Phys. Chem. B 107, 9964 (2003).

46. X. Zhang, M. A. Young, O. Lyandres, R. P. VanDuyne. J. Am. Chem. Soc. 127, 4484 (2005).

47. J. Kneipp, H. Kneipp, K. Kneipp. Chem. Soc. Rev. 37, 1052 (2008).

48. C. H. John, P. V. D. Richard. J. Vac. Sci. Technol., A 13, 1553 (1995).

49. D. J. Sirbuly, A. Tao, M. Law, R. Fan, P. Yang. Adv. Mater. 19, 61 (2007).

50. A. R. Tao, P. Sinsermsuksakul, P. Yang. Angew. Chem., Int. Ed. 45, 4597 (2006). 